\title{
Evaluation of real-time PCR for Strongyloides stercoralis and hookworm as diagnostic tool in asymptomatic schoolchildren in Cambodia
}

Fabian Schär ${ }^{a, b}$, Peter Odermatt ${ }^{a, b}$, Virak Khieu ${ }^{a, b, c}$, Marcus Panning ${ }^{e}$, Socheat Duong ${ }^{c}$, Sinuon Muth ${ }^{\mathrm{c}}$, Hanspeter Marti ${ }^{\mathrm{b}, \mathrm{d}}$, Stefanie Kramme $\mathrm{e}^{\mathrm{b}, \mathrm{d}}$

a Department of Epidemiology and Public Health, Swiss Tropical and Public Health Institute, Basel, Switzerland

b University of Basel, Basel, Switzerland

c National Centre for Parasitology, Entomology and Malaria Control, Ministry of Health, Phnom Penh, Cambodia

d Department of Medical Services, Swiss Tropical and Public Health Institute, Basel, Switzerland

e Department of Virology, University Medical Centre, Freiburg, Germany

\section{Corresponding author:}

Dr. med. S. Kramme, Swiss Tropical and Public Health Institute, Socinstr. 57, P.O. Box, 4002 Basel, Switzerland, Tel. ++41-61 284 8244, Fax: ++41-61 284 8118, E-mail address: stefanie.kramme@unibas.ch

Dr. P. Odermatt, Swiss Tropical and Public Health Institute, Socinstr. 57, P.O. Box, 4002 Basel, Switzerland, Tel. ++41-61 284 8214, E-mail address: peter.odermatt@unibas.ch 


\section{Abstract}

Diagnosis of soil-transmitted helminths such as Strongyloides stercoralis and hookworms (Ancylostoma duodenale and Necator americanus) is challenging due to irregular larval and egg output in infected individuals and insensitive conventional diagnostic procedures. Sensitive novel real-time PCR assays have been developed. Our study aimed to evaluate the real-time PCR assays as a diagnostic tool for detection of Strongyloides spp. and hookworms in a random stool sample of 218 asymptomatic schoolchildren in Cambodia.

Overall prevalence of $17.4 \%$ (38/218) and $34.9 \%$ (76/218) were determined by real-time PCR for S. stercoralis and hookworms, respectively. Sensitivity and specificity of S. stercoralis specific real-time PCR as compared to the combination of Baermann/Koga Agar as gold standard were $88.9 \%$ and $92.7 \%$, respectively. For hookworm specific realtime PCR a sensitivity of $78.9 \%$ and specificity of $78.9 \%$ were calculated. Co-infections were detectable by PCR in $12.8 \%(28 / 218)$ of individuals.

Strongyloides stercoralis real-time PCR applied in asymptomatic cases showed a lower sensitivity compared to studies undertaken with symptomatic patients with the same molecular tool, yet it proved to be a valid supplement in the diagnosis of STH infection in Cambodia.

Keywords: Real-time PCR, Strongyloides stercoralis, hookworm, sensitivity 


\section{Introduction}

Soil-transmitted helminths (STH) are the cause of the most neglected diseases worldwide (Hotez et al., 2006). STH are prevalent in tropical and sub-tropical regions with high prevalence rates in areas with poor hygiene and sanitation. In particular S. stercoralis and the hookworms Necator americanus and Ancylostoma duodenale represent clinically relevant members of STH. Strongyloides stercoralis, an intestinal nematode, infects an estimated 30-100 million people worldwide (Bethony et al., 2006). In most cases it causes asymptomatic infections. However, severe and life-threatening infections known as hyper-infection syndrome may occur (Marcos et al., 2008). Chronic infection with the hookworm may cause anemia and protein deficiency in both adults and children (Stoltzfus et al., 1997).

Traditionally, diagnosis is accomplished by microscopic examination of stool samples and culture methods. However, in light infected individuals microscopy remains insensitive and culture is time consuming (Agrawal et al., 2009). Sensitivity considerably increases when more than one sample is analyzed (Knopp et al., 2008; Marti and Koella, 1993). Use of consecutive samples on up to four days is therefore recommended (Dreyer et al., 1996). Importantly, parasite density in the majority of S. stercoralis cases is low, and therefore it would be desirable to increase the sensitivity of diagnostic methods. Diagnosis of hookworm infection is not as challenging due to higher parasite concentrations. Complicating, multiple infections with different STH can occur rendering simultaneous detection of different helminths a useful expansion of diagnostic tests. Novel molecular methods have been described.() Verweij and colleagues recently presented data of a pilot study conducted in Ghana describing a novel S. stercoralis real-time polymerase chain reaction (PCR) and a novel hookworm specific real-time PCR (Verweij et al., 2007; Verweij et al., 2009). These assays displayed a high analytical 
sensitivity and proved their principle. We validated a described Strongyloides spp. and hookworm real-time PCR assay in asymptomatic Cambodian children using a gold standard of microscopy and culture techniques. 


\section{Materials and Methods}

\subsection{Ethical consideration}

The study was integrated into a larger study on S. stercoralis infection and risk factors approved by the ethics committee of the cantons of Basel-Stadt and Basel-Land (EKBB, number 21/09, dated 29 January 2009), Switzerland, and the National Ethics Committee for Health Research (NECHR, number 033, dated 20 March 2009), Ministry of Health, Cambodia. Children with a positive diagnostic result for $S$. stercoralis and hookworm by microscopy were treated with ivermectin $(200 \mu \mathrm{g} / \mathrm{kg}$ BW divided in two doses, over two days) and mebendazole (500 mg, single dose), respectively.

\subsection{Sample collection}

During a field study in the semi-rural province of Kandal, south of Phnom Penh in Cambodia in 2009, 501 stool samples were collected from children from four different schools. The stool samples were transported to the laboratory within 2 hours after collection and subsequently analyzed by Baermann (Baermann, 1917), Koga Agar (Koga et al., 1991) and Kato-Katz (Katz et al., 1972) standard techniques. Kato Katz slides were read starting 30 minutes after preparation, to allow clearing time for the hookworm eggs. Koga Agar slides were read after incubation at $28^{\circ} \mathrm{C}$ for 48 hours. In addition, an aliquot of stool was taken and immediately frozen at $-20^{\circ} \mathrm{C}$ and shipped to Basel, Switzerland, for further analysis. A total of 218 samples were randomly selected $(114 ; 52.3 \%$ male versus $104 ; 47.3 \%$ female, average age 10.6 years) and analyzed by real-time PCR assays.

\subsection{DNA extraction from stool samples}


For isolation of nucleic acids from stool samples the QIAmp DNA stool kit (Qiagen, Hilden, Germany) was used according to the manufacturer's instructions. $200 \mathrm{mg}$ of stool was used as sample input volume, elution volume was $200 \mu \mathrm{l}$.

\subsection{Real-time PCR assays}

Real-time-PCR assays for S. stercoralis, A. duodenale and N. americanus were done according to Verweij et al. with minor modifications (Verweij et al., 2007; Verweij et al., 2009). In brief, a $25 \mu \mathrm{l}$ monoplex-reaction contained $5 \mu \mathrm{l}$ DNA template, $12,5 \mu \mathrm{l}$ HotStarMastermix (Qiagen), $3 \mathrm{mM} \mathrm{MgCl} 2,400 \mathrm{nM}$ of each sense and antisense primer (TIB-Molbiol, Berlin, Germany) and $200 \mathrm{nM}$ of the respective probe (TIB-Molbiol). All probes were labelled with the dye FAM and BBQ as quencher. Thermal cycling on an ABI 7500 Realtime PCR System (Applied Biosystems, Weiterstadt, Germany) comprised denaturation at $95^{\circ} \mathrm{C}$ for $10 \mathrm{~min}$, followed by 40 cycles of $95^{\circ} \mathrm{C}$ for $15 \mathrm{~s}$ and $60^{\circ} \mathrm{C}$ for $30 \mathrm{~s}$. Fluorescence was measured during the $60^{\circ} \mathrm{C}$ step.

\subsection{Construction of DNA standards}

A 101 base pair (bp) real-time amplicon of the 18S rRNA gene of S. stercoralis, a $70 \mathrm{bp}$ fragment of the ITS-2 gene of $A$. duodenale and a $100 \mathrm{bp}$ fragment of the ITS-2 gene of N. americanus was ligated into pCR2.1 plasmid vector and cloned into Escherichia coli using the pCR 2.1 TOPO TA cloning kit (Invitrogen, Karlsruhe, Germany) as described (Panning et al., 2008). Resulting plasmids of the correct length were purified by means of a QIAprep Spin Miniprep kit (Qiagen) and DNA concentration was spectrophotometrically measured. Each of the plasmids was used as a run control at a concentration of 100 DNA copies/PCR reaction. No-template controls were included in each run. 


\subsection{PCR inhibition control}

Inhibition was monitored by spiking the $S$. stercoralis plasmid at a concentration of 100 copies/real-time PCR reaction in each duplicate sample.

\subsection{Cross reactivity panel}

DNA of the following parasites was tested with each real-time PCR to assess a possible cross reaction: A. duodenale, N. americanus, Trichuris trichiura, Ascaris suum, Trichinella spiralis, Toxocara canis, Wuchereria bancrofti, Loa loa, Dirofilaria immitis, Echinococcus granulosus, Schistosoma mansoni, S. stercoralis.

\subsection{Data analysis}

Results of parasitological and real-time PCR examinations were double-entered into EpiData version 3.1 (EpiData Association, Odense Denmark) and Microsoft Excel, respectively. After validation, analysis was performed with Stata version 10 (Stata Corporation, Texas USA).

S. stercoralis real-time PCR were compared with results of Baermann and Koga Agar methods (gold standard) of the same stool sample. Results of Kato-Katz technique were regarded as gold standard for the hookworm real-time PCR assays. Standard statistical techniques (including McNemar test) were applied where appropriate. A $p$-value of less than $5 \%$ was regarded as statistically significant. 


\section{Results}

All real-time PCR assays were optimized with respect to sensitivity and specificity. 10 DNA plasmid copies per reaction of $A$. duodenale, N. americanus, Trichuris trichiura, Ascaris suum, Trichinella spiralis, Toxocara canis, Wuchereria bancrofti, Loa loa, Dirofilaria immitis, Echinococcus granulosus, Schistosoma mansoni, S. stercoralis were detectable on a regular basis by each individual real-time PCR. No unspecific amplification with other related parasites was detectable (data not shown).

\subsection{Validity of S. stercoralis PCR}

Overall, real-time PCR detected Strongyloides DNA in 38 of 218 (17.4\%) samples whereas Baermann and Koga Agar combined detected S. stercoralis larvae in 41 of 218 (18.8\%) samples ( $p=0.851) .25$ of $38(65.8 \%)$ real-time PCR positive samples were also

positive by Baermann and/or Koga Agar methods (Table 1). Real-time PCR failed to demonstrate DNA in two Baermann/Koga Agar positive and in one Baermann positive patient. In 17 Koga Agar positive samples only 4 (23.5\%) were also positive by real-time PCR. Stool samples of 164 study participants were negative in all three methods. None of the sample contained substances inhibitory to real-time PCR as demonstrated by amplification of the spiked external inhibition control. Sensitivity and specificity of realtime PCR as compared to the combination of Baermann/Koga Agar methods as gold standard were $88.9 \%$ and $92.7 \%$, respectively (Table 1 ).

Ct-values obtained for the real-time PCR positive samples were compared between Baermann/Koga Agar positive samples, Baermann positive/Koga Agar negative samples, Baermann negative/Koga Agar positive and Baermann/Koga Agar negative samples, respectively. Ct-values were low in samples with parasitological positive results and high 
in samples with negative results: Mean and median Ct-values were 31.9, 38.2, 35.0 and 39.1 and $33.0,39.0,34.5$ and 39.0, respectively. A statistically significant difference $(p<0.05)$ was observed between Baermann/Koga Agar positive and Baermann positive only samples and for Baermann/Koga Agar positive and Baermann/Koga Agar negative samples $(p=0.012 ;$ Figure 1$)$.

\subsection{Validity of hookworm PCR}

A total of 76 of $218(34.9 \%)$ samples yielded a positive result by real-time PCR of which $52(23.9 \%)$ samples were positive by the Kato-Katz method $(p=0.016) .41$ of $52(78.8 \%)$ of the Kato-Katz positive samples were positive by real-time PCR. Interestingly, 35 of $218(16.1 \%)$ samples yielded a positive real-time PCR result without detection of eggs. Of note, 16 of these $35(45.7 \%)$ samples yielded a Kato-Katz positive result when two further stool samples of these children were examined. Finally, 131 of $218(60.1 \%)$ samples demonstrated to be negative by all methods used. The overall analysis yielded a sensitivity of $78.9 \%$ and specificity of $78.9 \%$ of real-time PCR.

Ct-values of $N$. americanus real-time PCR positive samples were lower in Kato-Katz positive (median Ct-value 36.5) than Kato-Katz negative samples (median Ct-value 37.0), however no statistical difference was recorded ( $p=0.17$ ). Ct-values of $A$. duodenale realtime PCR positive samples were not done due to its low prevalence.

Real-time PCR was used for the identification of hookworm species. A total of 56 of 76 (73.7\%) hookworm real-time PCR positive samples were positive for $N$. americanus specific real-time PCR only. In particular five of 76 (6.6\%) were positive for $A$. duodenale specific real-time PCR only and 15 of 76 (19.7\%) samples yielded positive results for both $N$. americanus and $A$. duodenale specific real-time PCR. 
Among the $56 \mathrm{~N}$. americanus-DNA positive samples half $(28,50.0 \%)$ were concomitantly positive by microscopy. Only 1 of 5 A. duodenale-DNA positive sample (20.0\%) was also positive by microscopy.

Twelve of $15(80.0 \%)$ samples with positive results for $N$. americanus and $A$. duodenalespecific real-time PCR were also positive by Kato-Katz (Table 2)

\subsection{Samples with multiple detections}

Strongyloides spp. and hookworm co-infections were detected in 26 of 218 (11.9\%) samples by classical parasitological methods. Twenty-three of these positive samples (88.5\%) yielded a real-time PCR positive result. In detail, $13(50.0 \%)$ yielded positive results by both real-time PCR for $S$. stercoralis and hookworms; 2 (7.7\%) were positive by S. stercoralis real-time PCR only; 8 (30.8\%) samples were positive by hookworm realtime PCR only; 3 (11.5\%) were negative by both real-time PCR.

Interestingly, 11 of 15 (73.3\%) Koga Agar positive samples for S. stercoralis, which tested negative by Strongyloides real-time PCR yielded a hookworm positive result in the Kato-Katz method. In addition, 4 of 11 (36.4\%) hookworm positive samples Strongyloides larvae were found in Koga Agar method. 


\section{Discussion}

STH have a significant impact on public health in affected countries (Bethony et al., 2006) (Olsen et al., 2009). Many parts of Southeast Asia are ideal ecological and socioeconomical settings for S. stercoralis and hookworms. Reports from Cambodia and Laos PDR showed prevalence rates as high as $20.2 \%$ for S. stercoralis (Chhakda et al., 2006) and $76.8 \%$ for hookworms (Sayasone et al., 2011). Therefore, precise and reliable diagnostic methods remain crucial for public health interventions. Recently, molecular methods have been established for Strongyloides spp. and hookworms.(Verweij et al., 2009). Using the S. stercoralis positive results of Baermann and/or Koga Agar, our validation of real-time detection of DNA for Strongyloides spp. and hookworms revealed a sensitivity and specificity of $61.0 \%$ and $92.7 \%$ and $78.9 \%$ and $78.9 \%$, respectively. These values for sensitivity of $S$. stercoralis detection are similar to the values obtained by Verweij and colleagues (61.1\% and $92.4 \%)(2007,2009)$.

When comparing the coprological diagnostic methods with the real-time PCR, different factors have to be considered. First, the sample input volume of culture methods and of real-time PCR assays differs by a factor of 10 , which considerably increases sensitivity for the culture method. Increasing input volume for real-time PCR is not an option since stool contains substances which may be inhibitory to real-time PCR. However, real-time PCR inhibition was not an issue in our samples as no significant inhibition of the external amplification control was observed. As reported earlier and confirmed in our study use of consecutive samples increased sensitivity by a factor of 1.5 for Strongyloides as well as for the hookworms by conventional methods (Marti and Koella, 1993). It is likely that repeated testing of consecutive sample by real-time PCR will also increase the detection rate. However, this is not an option in resource limited settings but might be considered in in-depth epidemiological studies. 
Second, in our study only asymptomatic patients were included. It is reasonable to assume that these patients do not excrete S. stercoralis larvae and hookworm eggs in high concentrations. Symptomatic infections are more likely to be of higher parasite burden, thus increasing the number of detectable cases. This is also reflected by the overall high Ct-values in our study indicating rather low parasite burden.

Technically, for maximum sensitivity we used a monoplex real-time PCR approach in contrast to Verweji et al. This further minimizes the risk of false-positive results as multiplex real-time PCR assays are prone to complex oligonucleotide/probe interactions. Analytical sensitivity, as demonstrated on dilution series of plasmid DNA, was comparable to other assays used for parasite detection. However, we can not rule out that infections with low parasite numbers were missed by this approach due to clumped occurrence (Poisson distribution) of the parasites. Interestingly, Wichmann and colleages (2009) have recently demonstrated that detection of free parasite DNA in serum is possible for Schistosoma spp. and might constitute a feasible alternative for pathogen detection (Wichmann et al., 2009). Given the life cycles of Strongyloides and hookworm infections this might also be a feasible approach and deserves further studies.

Although real-time PCR in particular seems to be a valid technique for diagnosis its application in resource poor settings remains limited due to rather expensive laboratory equipment. Koga Agar culture and/or Baermann technique can be performed at the village level in the field. They have yielded superior performance in this study. However, given the importance of multiple parasitic infections in endemic countries molecular techniques become more advantageous. Especially multiplex real-time PCR methods as proposed by Verweij and colleagues seem to be a valid and cost effective way in diagnosing multiple infections (Verweij et al., 2007). Of note, experience with microscopy and conventional methods is waning in many laboratories in industrialized countries. In 
this respect, for $S$. stercoralis sensitivity of real-time PCR in comparison to the Koga Agar method was disappointing. One reason might by a possible misidentification of larvae. Further studies are needed if molecular methods can compensate for this.

To conclude, we could demonstrate that real-time PCR assays can be applied in asymptomatic cases but sensitivity was slightly lower compared to culture and/or microscopy. Further studies are needed to determine the applicability of real-time PCR in the clinical as well as in a research setting. 


\section{References}

Agrawal, V., Agarwal, T., Ghoshal, U.C., 2009. Intestinal strongyloidiasis: a diagnosis frequently missed in the tropics. Transactions of the Royal Society of Tropical Medicine and Hygiene 103, 242-246.

Baermann, G., 1917. Eine einfache Methode zur Auffindung von Ankylostomum (Nematoden) Larven in Erdproben. Mededeel mit H. Geneesk Laboratories Weltevreden, Feestbundel, Batavia., 41-47.

Bethony, J., Brooker, S., Albonico, M., Geiger, S.M., Loukas, A., Diemert, D., Hotez, P.J., 2006. Soil-transmitted helminth infections: ascariasis, trichuriasis, and hookworm. Lancet $367,1521-1532$.

Chhakda, T., Muth, S., Socheat, D., Odermatt, P., 2006. Intestinal parasites in schoolaged children in villages bordering Tonle Sap Lake, Cambodia. The Southeast Asian journal of tropical medicine and public health $37,859-864$.

Dreyer, G., Fernandes-Silva, E., Alves, S., Rocha, A., Albuquerque, R., Addiss, D., 1996. Patterns of detection of Strongyloides stercoralis in stool specimens: implications for diagnosis and clinical trials. Journal of clinical microbiology 34, 2569-2571.

Hotez, P.J., Molyneux, D.H., Fenwick, A., Ottesen, E., Ehrlich Sachs, S., Sachs, J.D., 2006. Incorporating a rapid-impact package for neglected tropical diseases with programs for HIV/AIDS, tuberculosis, and malaria. PLoS medicine 3, e102.

Katz, N., Chaves, A., Pellegrino, J., 1972. A simple device for quantitative stool thicksmear technique in Schistosomiasis mansoni. Rev Inst Med Trop Sao Paulo 14, 397-400. 
Knopp, S., Mgeni, A.F., Khamis, I.S., Steinmann, P., Stothard, J.R., Rollinson, D., Marti, H., Utzinger, J., 2008. Diagnosis of soil-transmitted helminths in the era of preventive chemotherapy: effect of multiple stool sampling and use of different diagnostic techniques. PLoS neglected tropical diseases 2, e331.

Koga, K., Kasuya, S., Khamboonruang, C., Sukhavat, K., leda, M., Takatsuka, N., Kita, K., Ohtomo, H., 1991. A modified agar plate method for detection of Strongyloides stercoralis. The American journal of tropical medicine and hygiene 45, 518-521.

Marcos, L.A., Terashima, A., Dupont, H.L., Gotuzzo, E., 2008. Strongyloides hyperinfection syndrome: an emerging global infectious disease. Transactions of the Royal Society of Tropical Medicine and Hygiene 102, 314-318.

Marti, H., Koella, J.C., 1993. Multiple stool examinations for ova and parasites and rate of false-negative results. Journal of clinical microbiology 31, 3044-3045.

Olsen, A., van Lieshout, L., Marti, H., Polderman, T., Polman, K., Steinmann, P., Stothard, R., Thybo, S., Verweij, J.J., Magnussen, P., 2009. Strongyloidiasis--the most neglected of the neglected tropical diseases? Transactions of the Royal Society of Tropical Medicine and Hygiene 103, 967-972.

Panning, M., Kilwinski, J., Greiner-Fischer, S., Peters, M., Kramme, S., Frangoulidis, D., Meyer, H., Henning, K., Drosten, C., 2008. High throughput detection of Coxiella burnetii by real-time PCR with internal control system and automated DNA preparation. BMC microbiology 8,77 .

Sayasone, S., Mak, T.K., Vanmany, M., Rasphone, O., Vounatsou, P., Utzinger, J., Akkhavong, K., Odermatt, P., 2011. Helminth and intestinal protozoa infections, 
multiparasitism and risk factors in Champasack province, Lao People's Democratic Republic. PLoS neglected tropical diseases 5, e1037.

Stoltzfus, R.J., Dreyfuss, M.L., Chwaya, H.M., Albonico, M., 1997. Hookworm control as a strategy to prevent iron deficiency. Nutrition reviews 55, 223-232.

Verweij, J.J., Brienen, E.A., Ziem, J., Yelifari, L., Polderman, A.M., Van Lieshout, L., 2007. Simultaneous detection and quantification of Ancylostoma duodenale, Necator americanus, and Oesophagostomum bifurcum in fecal samples using multiplex real-time PCR. The American journal of tropical medicine and hygiene 77, 685-690.

Verweij, J.J., Canales, M., Polman, K., Ziem, J., Brienen, E.A., Polderman, A.M., van Lieshout, L., 2009. Molecular diagnosis of Strongyloides stercoralis in faecal samples using real-time PCR. Transactions of the Royal Society of Tropical Medicine and Hygiene 103, 342-346.

Wichmann, D., Panning, M., Quack, T., Kramme, S., Burchard, G.D., Grevelding, C., Drosten, C., 2009. Diagnosing schistosomiasis by detection of cell-free parasite DNA in human plasma. PLoS neglected tropical diseases 3, e422. 
Table 1: Comparison of results obtained by Baermann and Koga Agar and real-time PCR methods for the detection of Strongyloides stercoralis

\begin{tabular}{ccccccc}
\hline & Microscopy & & \multicolumn{2}{c}{ PCR } & Sensitivity & Specificity \\
$\mathbf{n}$ & Baermann & Koga Agar & Positive & Negative & $\%$ & $\%$ \\
\hline 18 & + & + & 16 & 2 & 88.9 \\
6 & + & - & 5 & 1 & 83.3 & \\
17 & - & + & 4 & 13 & 23.5 & \\
41 & $+/-$ & $+/-$ & 25 & 16 & 61.0 & \\
177 & - & - & 13 & 164 & & 92.7 \\
\hline
\end{tabular}


Table 2: $\quad$ Comparison of results obtained by Kato-Katz and real-time PCR methods for the detection of hookworms.

\begin{tabular}{cccccc}
\hline $\mathbf{n}$ & Microscopy & PCR & & Sensitivity & Specificity \\
& Kato-Katz & Positive & Negative & $\%$ & $\%$ \\
\hline 52 & + & 41 & 11 & 78.9 & \\
166 & - & 35 & 131 & & 78.9 \\
\hline
\end{tabular}


Figure 1: Comparison of Ct-values of Strongyloides stercoralis real-time PCR results with Baermann and/or Koga Agar positive and negative samples, respectively. Y-axis denotes Ct-values of real-time PCR.

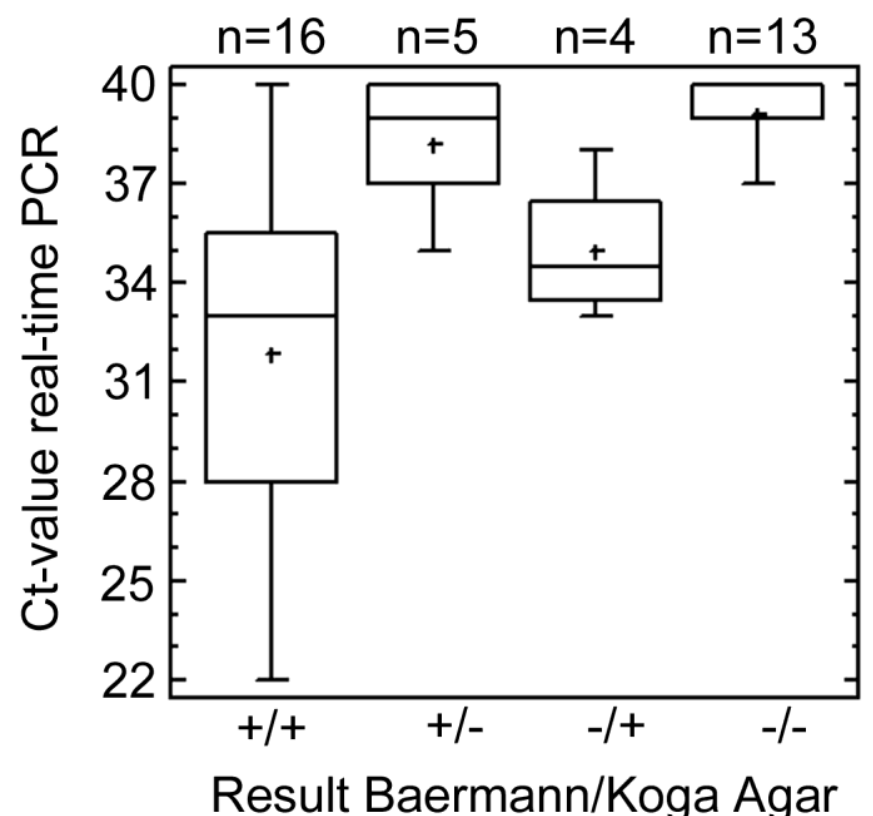

